Brief paper

\title{
Hand movement recognition based on biosignal analysis
}

\author{
Pawel Wojtczak ${ }^{a}$, Tito G. Amaral ${ }^{\text {b,c,* }}$, Octavio P. Dias ${ }^{\text {b,d }}$, Andrzej Wolczowski ${ }^{\text {, }}$, Marek Kurzynski ${ }^{\text {e }}$ \\ ${ }^{a}$ Faculty of Electronics, Wroclaw University of Technology, Wybrzeze Wyspianskiego 27, 50-370 Wroclaw, Poland \\ ${ }^{\mathrm{b}}$ Escola Superior de Tecnologia de Setubal, CESET, Instituto Politecnico de Setubal, Campus do IPS, Setubal, Portugal \\ c Institute of Systems and Robotics, University of Coimbra, 3030-290 Coimbra, Portugal \\ ${ }^{\mathrm{d}}$ INESC - Lisboa, Portugal \\ ${ }^{\mathrm{e}}$ Institute of Robotics and Informatics, Faculty of Electronics, Technical University of Wroclaw, Wroclaw, Poland
}

\section{A R T I C L E I N F O}

\section{Article history:}

Received 1 August 2008

Received in revised form

28 November 2008

Accepted 12 December 2008

\section{Keywords:}

Electromyography

Linear neural network

EMG signal classification

Prosthesis

Hand movement recognition

\begin{abstract}
A B S T R A C T
This paper proposes a methodology that analyses and classifies the electromyographic (EMG) signals using neural networks to control multifunction prostheses. The control of these prostheses can be made using myoelectric signals taken from surface electrodes. Finger motions discrimination is the key problem in this study. Thus the emphasis, in the proposed work, is put on myoelectric signal processing approaches. The EMG signals classification system was established using the linear neural network. The experimental results show a promising performance in classification of motions based on biosignal patterns.
\end{abstract}

(c) 2009 Elsevier Ltd. All rights reserved.

\section{Introduction}

Biomedical signal means a set of electrical signals acquired from any organ that represents a physical variable of interest. These signals are normally a function of time and can be analyzed in its amplitudes, frequency and phase. In the proposed method it is used biomedical signal-electromyographic (EMG) - to control the movement of prostheses.

Prosthesis systems for upper limb are mainly based on myoelectric control, recognizing EMG signals that occur during muscle contraction on the skin surface. Myoelectric control takes advantage of the fact that, after a hand amputation, great majority of the muscles that generate finger motion is left in the stump. The activity of these muscles still depends on the patient's will, so biosignals that occur during it, can be used to control prosthesis motion.

The control strategy of prostheses is based on to generate set of repeatable muscle contraction that is different from ordinary arm function. Contrary to conventional prosthesis control methods, it is possible to extract some feature from the myoelectric signals which may provide information about muscle activity below the skin. The extraction of features can be accomplished using various

\footnotetext{
* Corresponding author at: Escola Superior de Tecnologia de Setubal, CESET, Instituto Politecnico de Setubal, Campus do IPS, Setubal, Portugal.

E-mail addresses: pawel.wojtczak@student.pwr.wroc.pl (P. Wojtczak), tamaral@est.ips.pt (T.G. Amaral), pdias@est.ips.pt (O.P. Dias),

andrzej.wolczowski@pwr.wroc.pl (A. Wolczowski), marek.kurzynski@pwr.wroc.pl (M. Kurzynski).
}

techniques including signal amplitude, coefficients of EMG autoregressive (AR) model (Asres et al., 1996; Knox and Brooks, 1994), EMG frequency characteristic and power spectrum analyzed by fast Fourier transform (FFT) method (Kuribayachi et al., 1992; Hirawa et al., 1989), the integral of the absolute value (IAV) (Kuribayachi et al., 1993; Khoshaba et al., 1990), time and frequency histograms (Amaral et al., 2008).

In the proposed approach, an identification system will try to recognize a certain group of hand movements based on electrical signals (EMG signals) recorded on a patients forearm. The features used are based on time and energy histograms combined with a neural network based classification. The measurements were done on a specialized stand designed for such research.

\section{Measurement stand}

Measurement set was created specially for obtaining signals from patients arm. The configuration used in the measurement contained four input channels, which came from EMG sensors (Bagnoli-4 EM System User manual) acquiring analog myoelectric potentials from skin surface. Output is the file containing digital values of measured channels. For all measurements, videos are taken by digital camera allowing extracting data from specific stages of movement. General conception of acquisition system is presented in Fig. 1.

As shown on the schema, there are four main parts:

- AC card,

- galvanic separator,

0952-1976/\$ - see front matter (c) 2009 Elsevier Ltd. All rights reserved. doi:10.1016/j.engappai.2008.12.004 
- EMG sensors,

- digital camera.

Measurement set was equipped with $\mathrm{Ni} 4477$ card made by National Instruments (National Instruments, NI 4477 User Manual). The acquisition card has eight measurement channels with individual analog/digital converters (each has resolution 24 bits per channel) (Table 1 ).

The card was placed on PC board in a slot located as far as possible from others equipment (i.e. power supply, graphic card, hard disc, etc.). To provide additional noise protection special metal cage was prepared, applied and grounded. A very useful feature of the card is antialiasing filter that automatically adjusts to the desired frequency of sampling. This filter stifle signal in the band of $110 \mathrm{~dB}$, which helps eliminate frequency components above the Nyquist border (Wolczowski and Krysztoforski, 2002).

Detailed location of four EMG sensors is presented in Table 2. Those sensors were placed at the left hand. Two of them were situated close to the wrist and another two close to the elbow. One sensor from pair was attached to inner forearm side and second to outer side. Lines which determine distance from elbow and wrist are shown in Fig. 2.

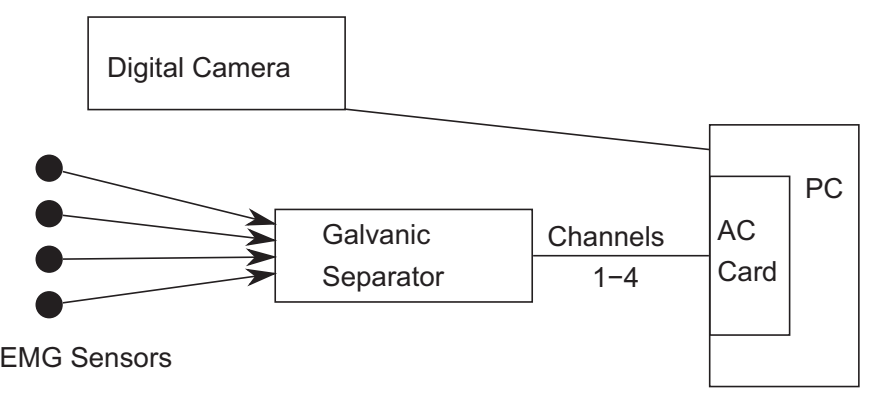

Fig. 1. Acquisition system.

Table 1

NI 4477 card characteristic.

\begin{tabular}{ll}
\hline Channels number & 8 \\
Analog input type & Bipolar, range $\pm 1-\mathrm{Vp}-\mathrm{p}$ \\
Input impedance & $1 \mathrm{MOhm}$ \\
Sampling resolution & $24 \mathrm{bits}, 1.19 \mu \mathrm{V}$ \\
Offset voltage & $\pm 3 \mathrm{mV}$ \\
Sampling frequency $f_{s}$ & $1-102.4 \mathrm{kHz}$ \\
Antialiasing filter & $\mathrm{DC}(0 \mathrm{~Hz})$ is \\
& $0.4535 f_{s}$ for passband \\
& $0.5465 f_{s}$ for stopband \\
& Damping $110 \mathrm{~dB}$ \\
\hline
\end{tabular}

Table 2

Channels used in the acquisition system.

\begin{tabular}{ll}
\hline Placement & Channel \\
\hline Inner side & \\
$\quad$ Near elbow & 1 \\
Near wrist & 2 \\
& \\
Outer side & 3 \\
Near elbow & \\
Near wrist & 4 \\
\hline
\end{tabular}

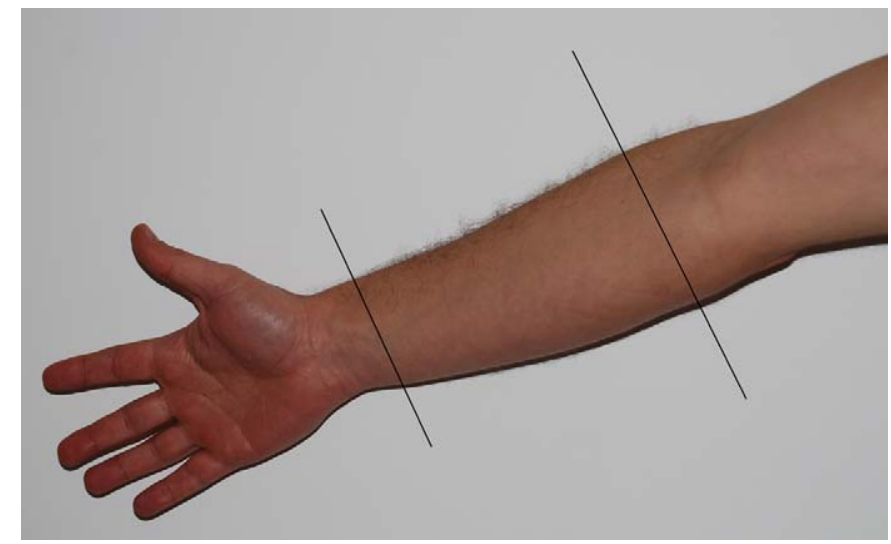

Fig. 2. Forearm sensors placement.

\section{Methodology description}

Using the acquisition system presented in the previous section, five files containing data were obtained. Each one include only one specified move repeated many times. To determine how many times and when exactly the movement took place it was essential to compare the data with the video. The video duration and the number of taken measurements are presented in Table 3.

The main control functions of the prosthesis are hand closing and opening, palmar flexion and dorsiflexion, wrist pronation and supination (Asres et al., 1996; Kuribayachi et al., 1992; Barrero et al., 2001). The list of grip movements used in the proposed work are:

1. palm (Fig. 3),

2. tip (Fig. 4),

3. cylindrical (high diagonal object) (Fig. 5),

4. cylindrical (small diagonal object) (Fig. 5),

5. spherical (Fig. 6).

\subsection{Data visualization and analysis}

Each file containing data were divided into smaller parts. Each part contains only one grasp movement shown in Fig. 7 which will be used in the feature extraction stage. In process of getting the feature vectors it is necessary to obtain the energy of each measurement and the corresponding number of signal crossing zero. In Fig. 8 the energy of a single grasp movement is illustrated.

Data were sampled with $1 \mathrm{KHz}$ frequency, so before getting feature vectors it was decided to apply $300 \mathrm{~Hz}$ lowpass Butterworth filter to eliminate same noises.

\subsection{Feature extraction}

Feature vector is twice the number of channels which is being analyzed. In this particular example there are four channels, which means that the length of feature vector is eight. From each channel are obtained the zero-crossing (ZC) number and the energy of the signal grasp movement defined by the following stages:

1. Calculate normalized number of zero-crossings in channel $i$ :

$$
Z C_{i}=\frac{z_{i}}{l_{i}}
$$


Table 3

Videos details

\begin{tabular}{llc}
\hline Move & Number of meas. & Video length \\
\hline 1st & 20 & $0: 57: 199$ \\
2nd & 20 & $1: 00: 759$ \\
3rd & 22 & $1: 26: 280$ \\
4 th & 25 & $1: 25: 799$ \\
5 th & 27 & $1: 32: 079$ \\
\hline
\end{tabular}

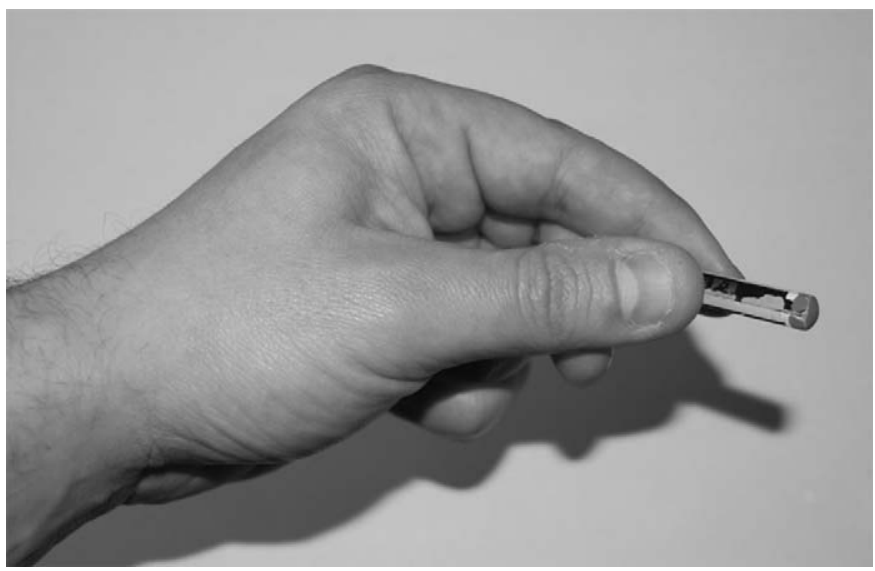

Fig. 3. Palm grip.

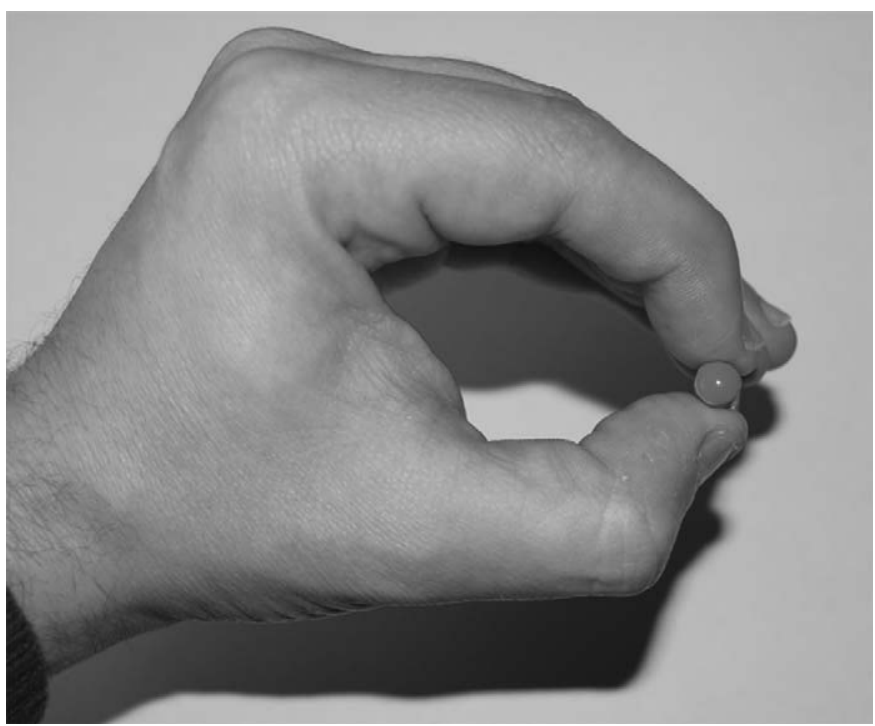

Fig. 4. Tip grip.

where $z_{i}$ is the number of zero-crossings in channel $i$ and $l_{i}$ is its length.

2. Calculate normalized sum of energy in channel $i$ :

$$
E_{i}=\frac{\sum_{k=1}^{l}\left|c_{i}(k)\right|}{l_{i}}
$$

where $c_{i}(k)$ is $k$ th absolute value in channel $i$ and $l_{i}$ is its length.

3. Repeat steps 1 and 2 for each channel of one measurement.

4. Divide all $Z C_{i}$ by the highest value of zero crossings from all measurements.

5. Divide all $E_{i}$ by the highest value of energy from all measurements.

6. Join all features in one feature vector, so for $n$ channels measurement the structure of the feature vector would be like the one presented in Table 4.

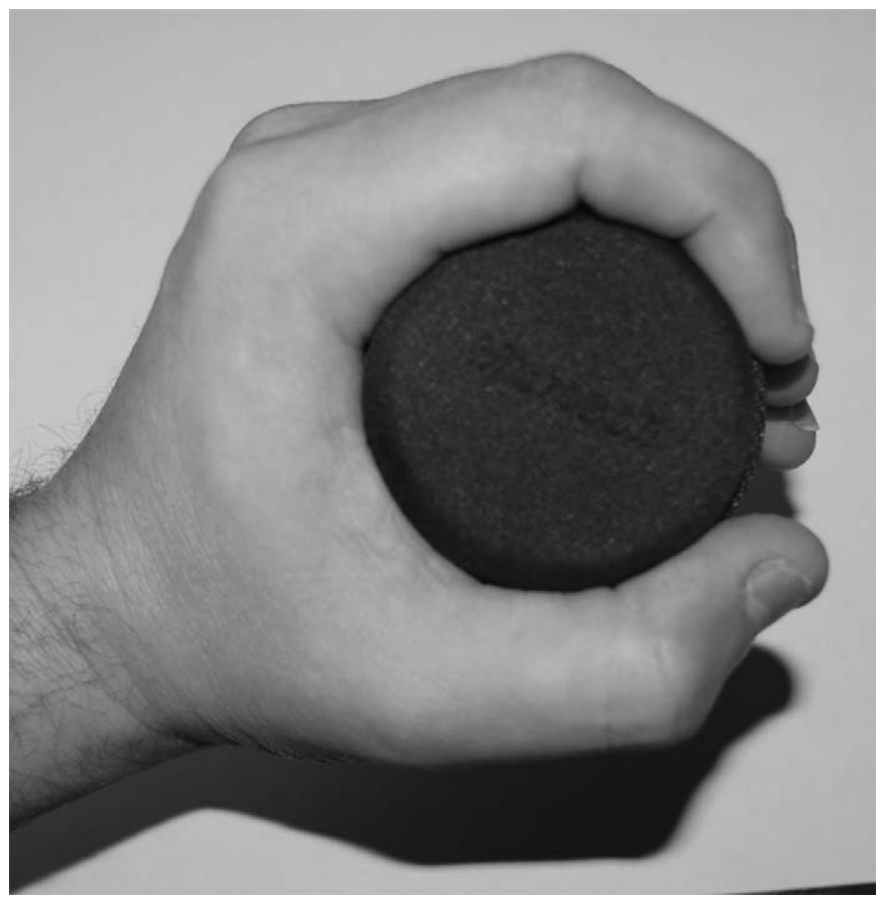

Fig. 5. Cylindrical grip.

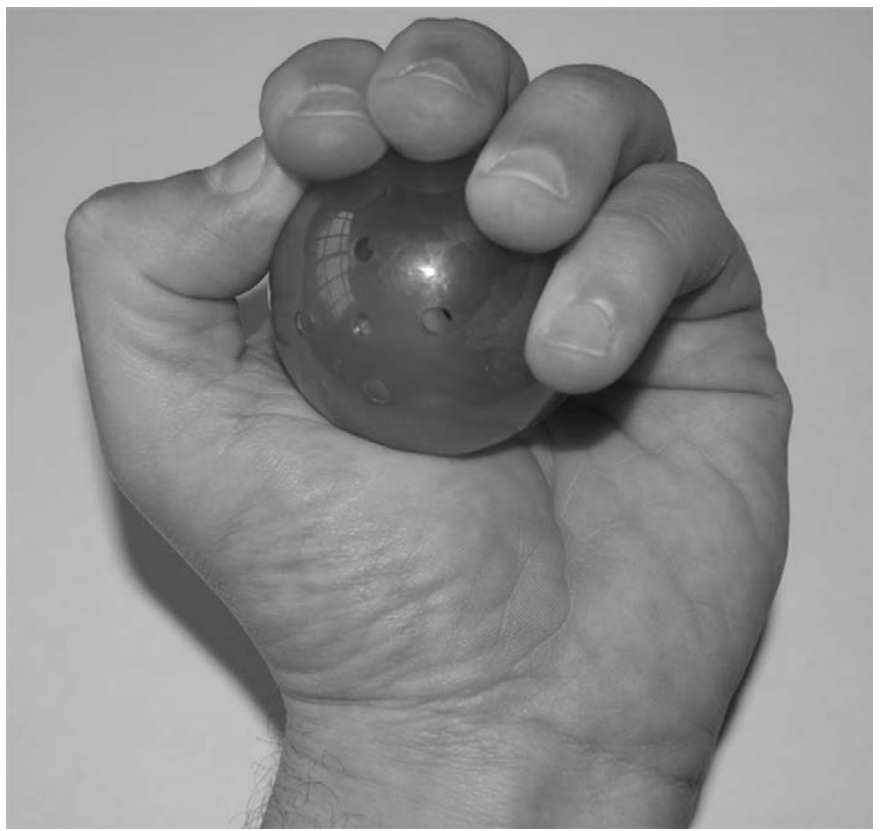

Fig. 6. Spherical grip.

The subscript digits in the zero crossing (ZC) and energy (E) values determine channel number.

Based on acquired data 114 feature vectors were obtained and divided in two groups: the learning and testing groups.

\subsection{Classification method}

The obtained feature vectors are used as inputs in the neural network. The output have the same length as the number of recognized moves and it is assumed that the highest value on output determine the type of grasp movement. 


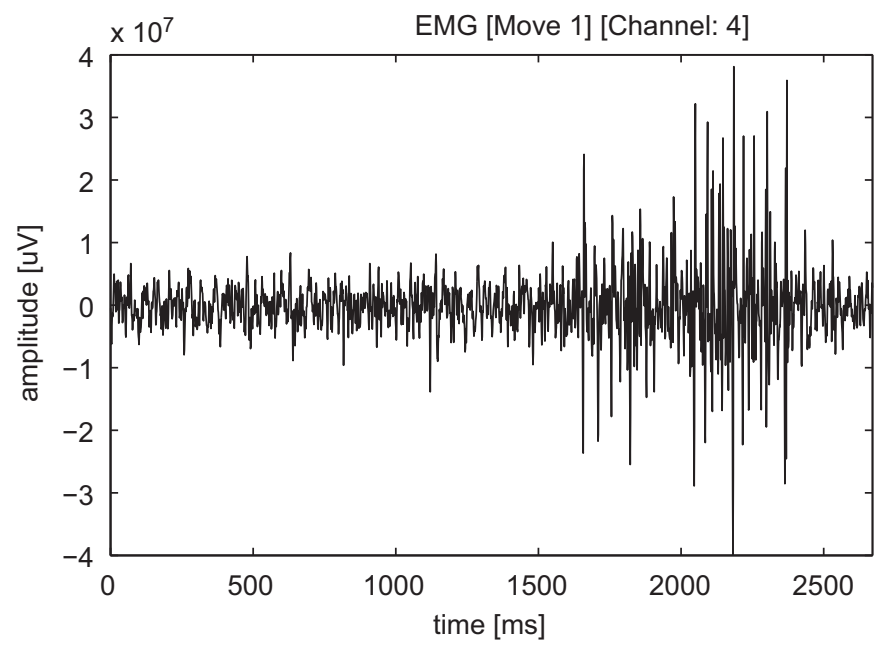

Fig. 7. Single move measurement.

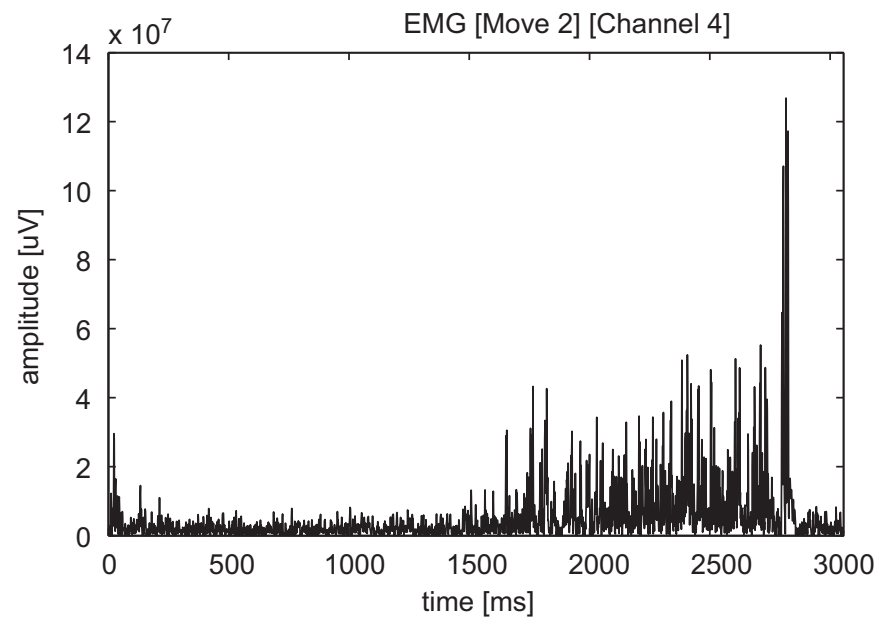

Fig. 8. Single move energy measurement.

Table 4

Feature vector.

\begin{tabular}{lllllll}
\hline 1 & 2 & 3 & 4 & $\cdots$ & $2 n-1$ & $2 n$ \\
\hline$Z C_{1}$ & $E_{1}$ & $Z C_{2}$ & $E_{2}$ & $\cdots$ & $Z C_{n}$ & $E_{n}$ \\
\hline
\end{tabular}

Fig. 9 presents the structure of the neural network used to analyze biosignals, where

- $p$-input feature vector,

- $R$-number of elements in input feature vector,

- $S$-length of output vector,

- $a$-output vector,

- $W$-matrix of weighs,

- $b$-bias.

Neural network used for classification had one five-neuron layer and the transfer function in each neuron was positive linear function:

$\operatorname{poslin}(x)= \begin{cases}0 & \text { if } x \leqslant 0 \\ x & \text { if } x>0\end{cases}$

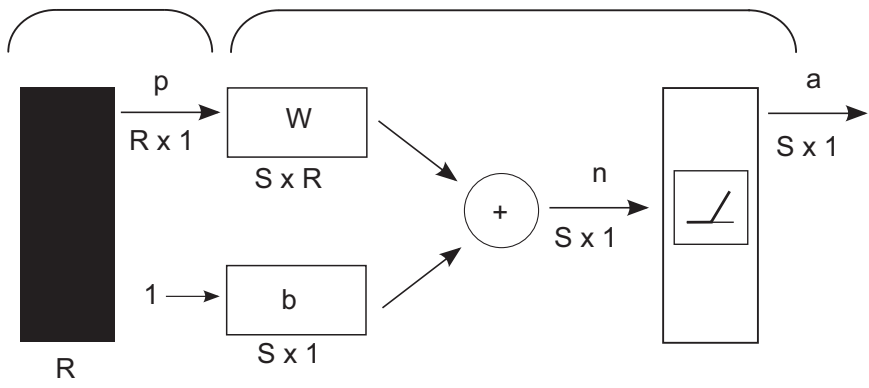

Fig. 9. Neural network structure.

The neural network output is expressed by

$a=\operatorname{poslin}(p \cdot W+b)$

where the weight matrix $W$ and biases vector $b$ were initiated with zeros. During the training weights and biases are updated with Widrow-Hoff learning function MATLAB:

$W(k+1)=W(k)+2 \alpha e(k) p^{\mathrm{T}}(k)$

$b(k+1)=b(k)+2 \alpha e(k)$

where $k$ is the iteration number; $\alpha$, the learning rate; $e$, the error vector; $p$, the input vector matrix. The error vector is obtained by the difference between target and neural network output:

$e_{q}(k)=t_{q}(k)-p_{q}(k)$

In the network learning rule it is provided a set of examples of desired network behavior:

$\left\{p_{1}, t_{1}\right\},\left\{p_{2}, t_{2}\right\}, \ldots,\left\{p_{q}, t_{q}\right\}, \ldots,\left\{p_{Q}, t_{Q}\right\}$.

The $p_{q}$ value is an input to the network, and $t_{q}$ value is the corresponding target output. As each input is applied to the network, the network output is compared to the target. The error is calculated as the difference between the target output and the network output. The goal is to minimize the objective function given by the average of the sum of these errors (9).

$m s e=\frac{1}{Q} \sum_{k=1}^{Q} e(k)^{2}=\frac{1}{Q} \sum_{k=1}^{Q}(t(k)-a(k))^{2}$

The least mean square (LMS) error algorithm adjusts the weighs and biases of the linear network so as to minimize these objective functions MATLAB.

\section{Experimental results}

For testing the biosignal recognition system 114 feature vectors were obtained from acquired data. All tests were performed in the Matlab running on a processor AMD Turion 64X2 Mobile $2 \times 800 \mathrm{MHz}$ with $1024 \mathrm{MB}$ of physical memory.

Vectors were used to train and test linear neural network. While the number of learning and testing vectors is similar the classification results are quite good, but when the ratio between the number of testing and learning vectors is growing, overall classification accuracy is decreasing (Fig. 10). These happens because there is a lost of representative samples in the population of the learning data set.

\subsection{Data divided into two sets}

When all the 114 features vectors were divided into two sets (learning and testing) the experimental result was $100 \%$ overall 


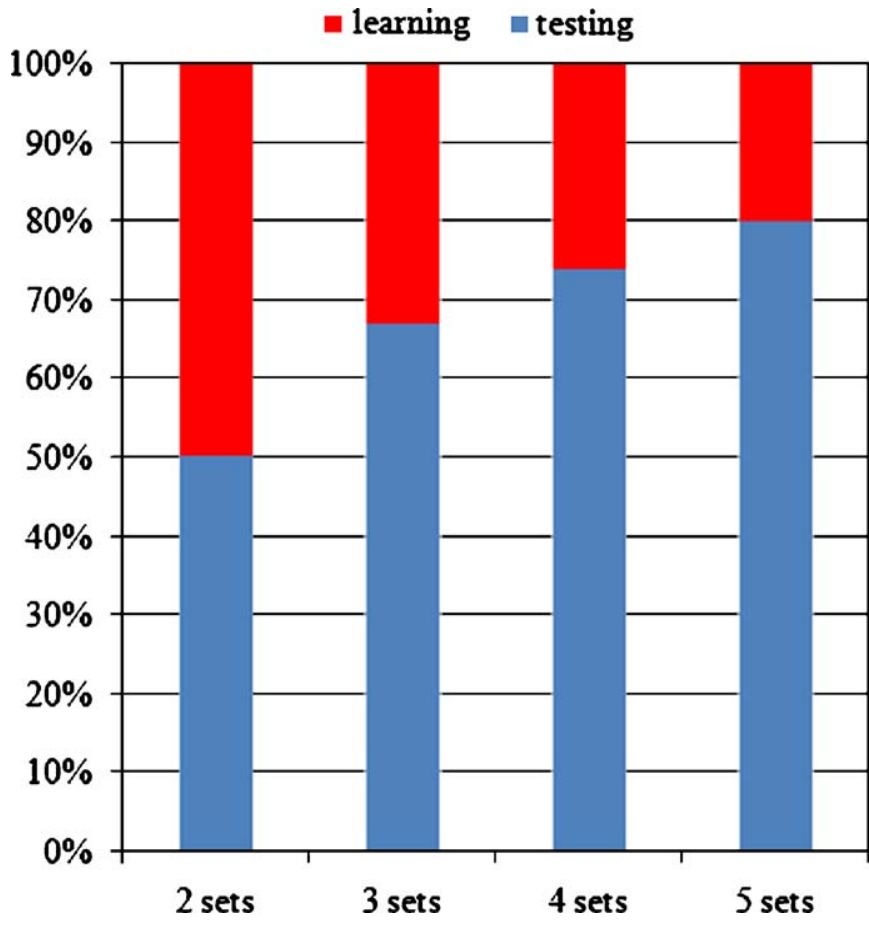

Fig. 10. Learning/testing vectors ratio.

Table 5

Results (2 sets).

\begin{tabular}{llllll}
\hline \multicolumn{5}{l}{ Move number } \\
\cline { 2 - 6 } & 1 & 2 & 3 & 4 & 5 \\
\hline Learn/test & $10 / 10$ & $10 / 10$ & $11 / 11$ & $13 / 12$ & $13 / 14$ \\
Correct/wrong & $10 / 0$ & $10 / 0$ & $11 / 0$ & $12 / 0$ & $14 / 0$ \\
{$[\%]$} & 100 & 100 & 100 & 100 & 100 \\
\hline
\end{tabular}

classification accuracy (Table 5). This result was obtained independently whose set was choose for testing and for learning.

Training performance goal was set to 0.025 , learning rate coefficient was set to 0.03 and average training network time was 1.72 seconds. Neural network training process is shown in Fig. 11. The same training performance was obtained choosing the training vector as testing vector and vice versa.

\subsection{Data divided into three sets}

In this experience, the data were divided into three sets, each contains similar number of each type vectors with a difference between quantity equal to or less than 1 vector. The number of testing vectors is approximately twice the number of learning vectors.

Training performance was set to 0.02 , learning rate coefficient was set to 0.03 and average training network time was 1.89 seconds. Neural network training process is shown in Fig. 12. The difference in the training process between the three possible sets of learning feature vectors is the epoch's number necessary to reach the training performance goal. This goal was obtained in the three experiences, approximately, after 475, 1050 and 1300 epochs.

The obtained result was $95.62 \%$ overall classification accuracy choosing in each experimental one of the three possible learning vectors (Tables 6-8). The 3rd and 4th type movements were

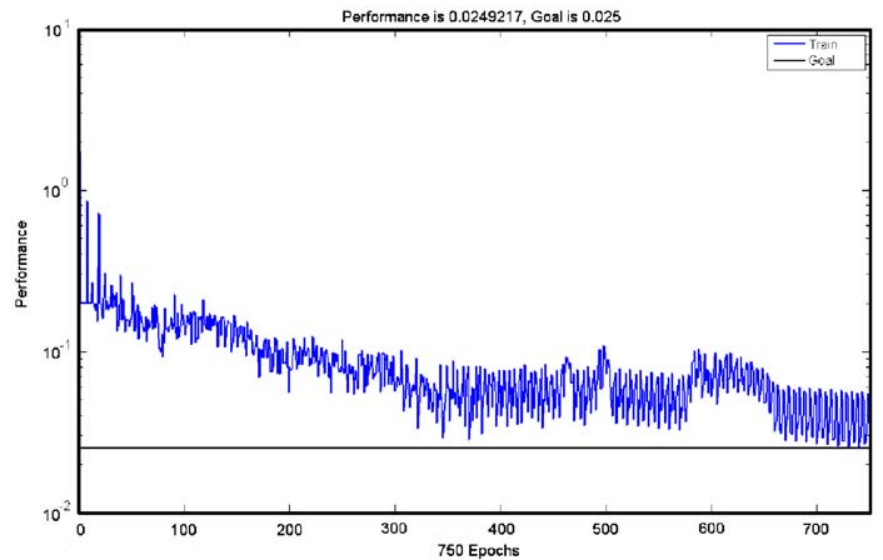

Fig. 11. Training of the neural network (set I).

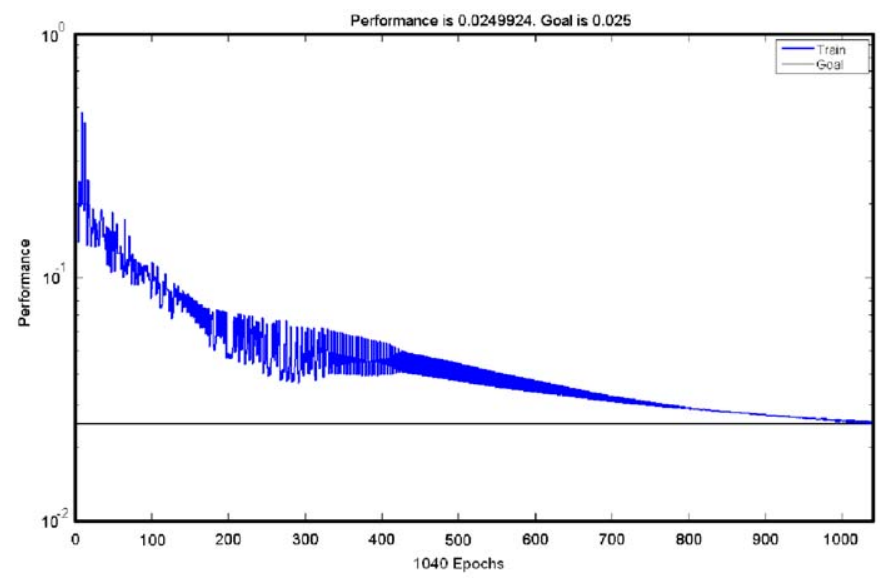

Fig. 12. Training of the neural network (set I).

Table 6

Classification set I.

\begin{tabular}{lrrrrr}
\hline Move & 1st & 2nd & 3rd & 4th & 5 th \\
\hline 1st & 13 & 0 & 0 & 0 & 0 \\
2nd & 0 & 12 & 1 & 0 & 0 \\
3rd & 0 & 0 & 15 & 0 & 0 \\
4th & 0 & 0 & 0 & 17 & 0 \\
5th & 0 & 0 & 0 & 0 & 18 \\
\hline
\end{tabular}

perfectly recognized, but the other moves were incorrectly classified, but only up to two times.

\subsection{Data divided into four sets}

In the following experience, the data were divided into four sets, each contains similar number of each type vectors also with a difference between quantity equal to or less than 1 .

Training performance was set to 0.02 , learning rate coefficient was set to 0.03 and average training network time was 2.03 seconds. Neural network training process is shown in Fig. 13.

The obtained result was $93.81 \%$ overall classification accuracy choosing in each experience one of the four possible learning vectors (Tables 9-12). The 3rd type movement was the most difficult to recognize, because mistake was taken five times in all the four experiences. Only the 4 th and 5 th moves were recognized without mistakes in the four tests. 
Table 7

Classification set II.

\begin{tabular}{lrrrrr}
\hline Move & 1st & 2nd & 3rd & 4 th & 5 th \\
\hline 1st & 13 & 0 & 0 & 0 & 0 \\
2nd & 0 & 13 & 1 & 0 & 0 \\
3rd & 0 & 0 & 14 & 0 & 0 \\
4th & 0 & 0 & 0 & 17 & 0 \\
5th & 0 & 0 & 1 & 0 & 17 \\
\hline
\end{tabular}

Table 8

Classification set III.

\begin{tabular}{lrrrrr}
\hline Move & 1st & 2nd & 3rd & 4 th & 5th \\
\hline 1st & 13 & 0 & 1 & 0 & 0 \\
2nd & 0 & 12 & 1 & 0 & 0 \\
3rd & 0 & 0 & 15 & 0 & 0 \\
4th & 0 & 0 & 0 & 16 & 0 \\
5th & 0 & 0 & 0 & 0 & 18 \\
\hline
\end{tabular}

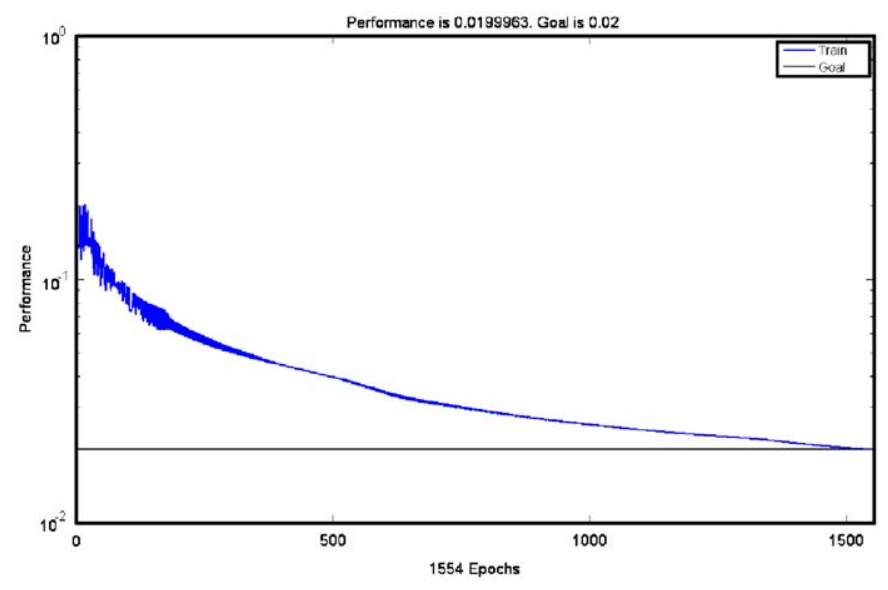

Fig. 13. Training of the neural network (set I).

Table 9

Classification set I.

\begin{tabular}{lrrrrr}
\hline Move & 1st & 2nd & 3rd & 4 th & 5 th \\
\hline 1st & 15 & 0 & 0 & 0 & 0 \\
2nd & 0 & 15 & 0 & 0 & 0 \\
3rd & 0 & 0 & 16 & 0 & 0 \\
4th & 0 & 0 & 0 & 19 & 0 \\
5th & 0 & 0 & 0 & 0 & 20 \\
\hline
\end{tabular}

\subsection{Data divided into five sets}

In the last experience, the data were divided into five sets, each containing a similar number of type vectors and also with a difference between quantity equal to or less than 1 . Training performance was set to 0.02 , the learning rate coefficient was set to 0.03 and the average training network time was 2.10 seconds. Neural network training process is shown in Fig. 14. The only difference in the training process between the five possible sets of learning feature vectors was the epochs number necessary to reach the training performance goal.
Table 10

Classification set II.

\begin{tabular}{lrrrrr}
\hline Move & 1st & 2nd & 3rd & 4 th & 5 th \\
\hline 1st & 15 & 0 & 0 & 0 & 0 \\
2nd & 0 & 15 & 0 & 0 & 0 \\
3rd & 1 & 1 & 14 & 0 & 0 \\
4th & 0 & 0 & 0 & 19 & 0 \\
5th & 0 & 0 & 0 & 0 & 20 \\
\hline
\end{tabular}

Table 11

Classification set III.

\begin{tabular}{lrrrrr}
\hline Move & 1st & 2nd & 3rd & 4 th & 5 th \\
\hline 1st & 15 & 0 & 0 & 0 & 0 \\
2nd & 0 & 14 & 1 & 0 & 0 \\
3rd & 1 & 0 & 16 & 0 & 0 \\
4th & 0 & 0 & 0 & 18 & 0 \\
5th & 0 & 0 & 0 & 0 & 21 \\
\hline
\end{tabular}

Table 12

Classification set IV.

\begin{tabular}{lrrrrr}
\hline Move & 1st & 2nd & 3rd & 4 th & 5th \\
\hline 1st & 14 & 0 & 0 & 1 & 0 \\
2nd & 0 & 15 & 0 & 0 & 0 \\
3rd & 1 & 1 & 15 & 0 & 0 \\
4th & 0 & 0 & 0 & 19 & 0 \\
5th & 0 & 0 & 0 & 0 & 20 \\
\hline
\end{tabular}

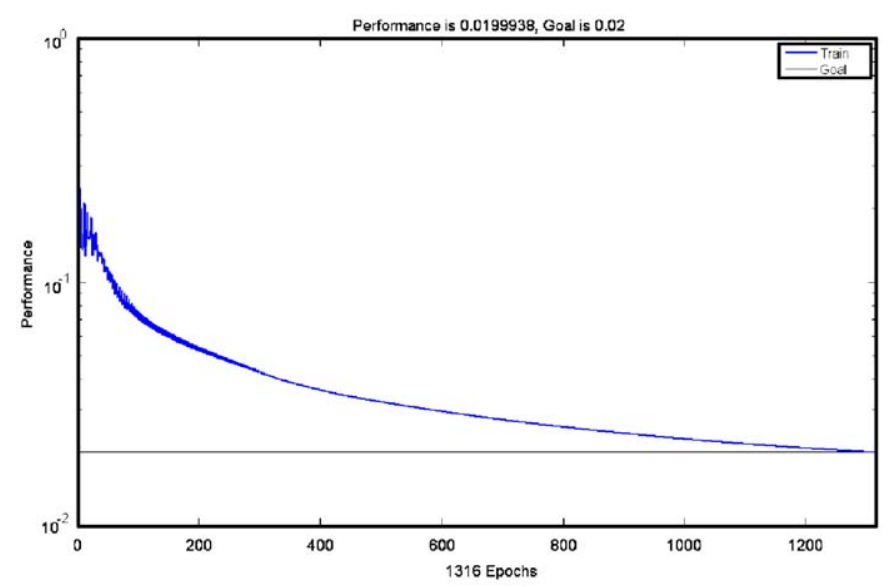

Fig. 14. Training of the neural network (set I).

The obtained result was $90.43 \%$ overall classification accuracy choosing in each experience one of the five possible learning vectors (Tables 13-17). In this case only 4 th and 5 th moves were always correctly recognized. The worst recognition was observed with 3rd type movement with five recognition faults.

Comparison results of different movement recognition techniques obtained in other research works are presented in Table 18 (Reaz et al., 2006). In the proposed work, the used of time and energy histograms as input features in the neural network based classification obtained an $100 \%$ of accuracy rate. In this case the set feature vectors were divided in the middle creating the learning and testing vectors sets. 
Table 13

Classification set I.

\begin{tabular}{lrrrrr}
\hline Move & 1st & 2nd & 3rd & 4 th & 5 th \\
\hline 1st & 16 & 0 & 0 & 0 & 0 \\
2nd & 0 & 15 & 1 & 0 & 0 \\
3rd & 0 & 0 & 17 & 0 & 0 \\
4th & 0 & 0 & 0 & 20 & 0 \\
5th & 0 & 0 & 0 & 0 & 22
\end{tabular}

Table 14

Classification set II.

\begin{tabular}{lrrrrr}
\hline Move & 1st & 2nd & 3rd & 4 th & 5 th \\
\hline 1st & 16 & 0 & 0 & 0 & 0 \\
2nd & 0 & 15 & 1 & 0 & 0 \\
3rd & 0 & 0 & 17 & 0 & 0 \\
4th & 0 & 0 & 0 & 20 & 0 \\
5th & 0 & 0 & 0 & 0 & 22 \\
\hline
\end{tabular}

Table 15

Classification set III.

\begin{tabular}{lrrrrr}
\hline Move & 1st & 2nd & 3rd & 4 th & 5th \\
\hline 1st & 15 & 0 & 0 & 1 & 0 \\
2nd & 0 & 16 & 0 & 0 & 0 \\
3rd & 1 & 1 & 16 & 0 & 0 \\
4th & 0 & 0 & 0 & 20 & 0 \\
5th & 0 & 0 & 0 & 0 & 21 \\
\hline
\end{tabular}

Table 16

Classification set IV.

\begin{tabular}{lrrrrr}
\hline Move & 1st & 2nd & 3rd & 4th & 5 th \\
\hline 1st & 15 & 0 & 0 & 1 & 0 \\
2nd & 0 & 15 & 0 & 0 & 1 \\
3rd & 1 & 1 & 14 & 0 & 0 \\
4th & 0 & 0 & 0 & 20 & 0 \\
5th & 0 & 0 & 0 & 0 & 22 \\
\hline
\end{tabular}

If the learning data set of a neural network contain representative information of the hand movement then the test results will be better than those obtained if it misses some important information in the learning data set.

In these studies, the training process and classification results of the fuzzy system gives more consistent classification results and is insensitive to over-training (Cheron et al., 1996). Normally, the coefficients of an EMG AR model are used as input features in a classification.

The use of the neural network techniques are very suitable for EMG signal recognition tasks where the variety and separability of the EMG patterns can be very complicated. The main difficulty with the use of the EMG signals is in the continuous control of prostheses where it is presented the non-linearity and nonstationarity characteristics of the EMG sensor information (Kristin et al., 1996). However, since the neural networks can acquire nonlinear mapping of data, it has also been applied in the prothesis control of other parts of the human body namely in the ankle-foot (Au et al., 2005), in the lower limb (Lee and Lee, 2005) or in the hand (Kristin et al., 1996).
Table 17

Classification set V.

\begin{tabular}{lrrrrr}
\hline Move & 1st & 2nd & 3rd & 4 th & 5th \\
\hline 1st & 16 & 0 & 0 & 0 & 0 \\
2nd & 0 & 15 & 0 & 0 & 0 \\
3rd & 1 & 0 & 17 & 0 & 0 \\
4th & 1 & 0 & 0 & 19 & 0 \\
5th & 0 & 0 & 0 & 0 & 22 \\
\hline
\end{tabular}

Table 18

Typical EMG classification accuracy rate.

\begin{tabular}{ll}
\hline Method & Accuracy rate (\%) \\
\hline Coefficient of AR & 99 \\
Neural networks & 84 \\
Fuzzy system & 85 \\
\hline
\end{tabular}

\section{Conclusion}

We have shown that a simple linear neural network can determine with great accuracy, of which five movements were performed. Furthermore, this was accomplished using only the normalized RMS power from each of the four sensors placed around the forearm. Use of different features and classifiers will undoubtedly improve the classification of the existing signals and allow classification on additional categories of hand kinematics.

The decomposition of each movement type in a set of individual movements will be one of the future directions of study.

\section{References}

Amaral, T., Dias, O., Prociow, P., Wolczowski, A., Filipe, J., 2008. Identification of hand movements based on MMG and EMG signals. In: Proceedings of the International Conference on Bio-inspired Systems and Signal Processing, pp. 534-539.

Asres, A., Dou, H.F., Zhou, Z.Y., Zhang, Y.L., Zhu, S.C., 1996. A combination of ar and neural network technique for EMG pattern identification. In: Proceedings of the Annual International Conference on IEEE Engineering in Medicine and Biology Society, vol. 4, pp. 1464-1465.

Au, S.K., Bonato, P., Herr, H., 2005. An EMG-position controlled system for an active ankle-foot prothesis: an initial experimental study. In: Proceedings of the International Conference on Rehabilitation Robotics, pp. 375-379.

Bagnoli-4 EM System User manual, 2003, DELSYS 〈www.delsys.com〉.

Barrero, V., Grisales, V., Rosas, F., Sanchez, C., Leon, J., 2001. Design and implementation of an intelligent interface for myoelectric controlled prosthesis. In: Proceedings of the IEEE International Conference on Engineering in Medicine and Biology Society, vol. 4, pp. 3352-3355.

Cheron, G., Draye, J-P., Bourgeios, M., 1996. A dynamic neural network identification of electromyography and trajectory relationship during complex movements. IEEE Trans. Biomed. Eng. 43, 552-558.

Hirawa, A., Shimohara, K., Tokunaga, Y., 1989. EMG pattern analysis and classification by neural network. In: Proceedings of the IEEE International Conference on IEEE Systems, Man and Cybernetics, vol. 3, pp. 1113-1115.

Khoshaba, T., Badie, K., Hashemi, R.M., 1990. EMG pattern classification based on back propagation neural network for prosthesis control. In: Proceedings of the IEEE International Conference on Engineering in Medicine and Biology Society, pp. 1474-1475.

Knox, R., Brooks, D.H., 1994. Classification of multifunction surface EMG using advanced ar model representations. In: Proceedings of the Annual Conference on Northeast Bioengineering, pp. 96-98.

Kristin, A.F., Ian, D.W., Richard, G.B., 1996. Myoelectric teleoperation of a complex robotic hand. IEEE Trans. Robotics Autom. 12, 775-787.

Kuribayachi, K., Okimura, K., Taniquichi, T., 1992. A discrimination system using neural network for EMG-controlled prostheses. In: Proceedings of the International Conference on IEEE Robot and Human Communication, pp. 63-68.

Kuribayachi, K., Shimuzu, S., Okimura, K., Taniquichi, T., 1993. A discrimination system using neural network for EMG-controlled prostheses-integral type of EMG signal processing. In: Proceedings of the IEEE International Conference on Intelligent Robots and Systems, pp. 1750-1755. 
Lee, J.-W., Lee, G.-K., 2005. Gait angle prediction for lower limb orthotics and prostheses using an EMG signal and neural networks. Int. J. Control Autom. System 3, 152-158.

Mathwork Matlab 2006a. Documentation, 2006. Natick, MA, Mathworks 〈www.mathworks.com $\rangle$.

National Instruments, NI 4477 User Manual, 2003 〈www.ni.com〉.
Reaz, M.B.I., Hussain, M.S., Yasin, M.F., 2006. Techniques of EMG Signal Analysis: Detection, Processing, Classification and Applications, Biological Procedures Online.

Wolczowski, A., Krysztoforski, K., 2002. Control-measurement circuit of myoelectric prosthesis hand. 4 (Supplement 1), 576-577; Proceedings of 13th Conference of the European Society of Biomechanics, Wroclaw, Poland September, 2002

Please cite this article as: Wojtczak, P., et al., Hand movement recognition based on biosignal analysis. Engineering Applications of Artificial Intelligence (2009), doi:10.1016/j.engappai.2008.12.004 